or strain of insulating materials of dielectrics in an electric field of force. Maxwell's theory led in the end to the invention of radio, and brought the antipodes within reach of a telephone call. But even Maxwell, though he had found by mathematical analysis the velocity with which electromagnetic waves travel in space, that is, the velocity of light, and knew that both the electric and magnetic fields in these waves were transverse oscillations of the ether at right angles to one another, did not find or state the law connecting them and the mode of transmission of their energy. This was done some twenty years later by Prof. J. H. Poynting who, in extending Maxwell's theory, derived the simple and powerful relation known as Poynting's theorem or law. It is that everywhere in Nature, energy flows by the mutual reaction of electric and magnetic fields, that it is delivered in a direction at right angles to the plane containing both of them, and that the power is simply proportional to the product of the fields. The passage of every form of electrical or mechanical energy obeys this law, and now that we know that there is nothing in the material universe but electricity, it may even be regarded as one of the most illuminating physical relations in Nature, since electricity or electrification moves everywhere accord. ing to this rule.

A field of force in physics is defined as a state of stress that is in its origin electric or magnetic. It produces attraction between unlike charges or poles and repulsion between those of the same signs. A short account was given of Rutherford's electrical theory of the constitution of matter, pointing out that in the metal of a 2,000-ton ship there are a little more than a ton of electrons. For energy to travel there must be in every case, electrical or mechanical, an electric field and a magnetic field at right angles to it. Around and in an electric wire carrying a current there are two electric fields, one outwards or inwards at right angles to its surface through the insulation around the wire, and the other along the conductor commonly called the ohmic drop.

Modern high-tension cables have a cylindrical sheath of solid insulation of very high quality round the conductors which can easily carry an electric field of 10,000 volts per $\mathrm{cm}$. This intense concentration of power is not in the wire but in the paper or rubber insulation surrounding it. Any electromagnetic energy that enters a metal never comes out of it except as heat, or in the case of machines, as mechanical stress.

At the end of a line where power is utilized there must be a relaxation of the electric field in order that energy may be transferred. There is no difference in principle between direct and alternating current transmission, for in the latter case both the electric and magnetic fields reverse together and the direction of the energy path does not change with that of the current. But if there is a difference in phase between the current and the voltage we have to multiply their product by the cosine of the phase angle to get the true power. Radio-transmission plays a large part in modern life and though the means by which it is done are highly technical and complicated, the movement of energy in free space once it has left the transmitter is relatively simple. The great masts and aerials that we see are in effect condensers to which very rapidly alternating voltage is applied. While a vertical antenna is being charged, in the millionth of a second, the current is moving upwards against the electric field that is piling up at the top, the direction of which is downwards.

When we come to consider the movement of energy in mechanical systems of transmission, we encounter the release of energy known as combustion. Sir William Ramsay was one of the first of chemists to realize the electrical nature of atomic forces.

Prof. Thornton concluded by carrying the general argument to its logical end. Before the coming of the electrical theory of matter, the highest flight of scientific imagination was to conceive a dead universe of cold worlds; but now we know that all that is necessary to bring it to an end, to uncreate it, is that the equal number of positive and negative charges that make up its atoms must be made to cancel one another out and 'leave not a rack behind'. But the energy that formed the universe will still remain, eternal and unchanged.

\title{
VISIBILITY AND ROAD ACCIDENTS
}

$\mathrm{M}$ OST research work on road accidents has been concerned with the physical or mental state of the driver of the car, but H. H. Ferguson and W. R. Geddes, in Occupational Psychology (14, No. 4), describe some recent work done in New Zealand on road accidents from a very unusual point of view. These writers investigated the beliefs of pedestrians regarding their own visibility, to see how far these beliefs compared with their actual visibility.

The method in outline was to get the subjects to walk along a straight, level, unlighted, open-country, dustless road, away from, or towards, the headlights of a stationary car. They had to indicate by pegs those points along the road at which they believed: (a) that it was just possible, but not likely, that they were out of the range of the visibility for the driver ; $(b)$ that they had a normal degree of certainty that they were just beyond the range of visibility for the driver ; (c) that they were certain of being just outside the range of visibility for the driver.
It had been discovered that the greatest distance at which a typically attired moving pedestrian could be distinguished as a pedestrian with reasonable certainty was approximately $320 \mathrm{ft}$., while beyond $463 \mathrm{ft}$. nothing could be seen of the pedestrian.

When the results were analysed a wide range of distribution of distances was revealed. A considerable number of the subjects considered themselves to be visible when actually well beyond the range of visibility; one subject thought he was visible at $814 \mathrm{ft}$.

The authors hold that, if these findings were to be substantiated by further research, publicity should be given to this possibility of error, for the belief that one is visible when one is actually invisible is a dangerous belief. This is an interesting contribution to the complex problem of road accidents. If this possibility of error is important in New Zealand it is still more important in Great Britain, and particularly in black-out conditions. 